\title{
Decreasing betatrophin levels during pregnancy in healthy women
}

A. Zielińska, R. Maciulewski, K. Siewko, A. Popławska-Kita, D. Lipińska, G. Kozłowska, M. Górska, M. Szelachowska

Department of Endocrinology, Diabetology and Internal Medicine; Medical University of Bialystok, Poland

\section{BACKGROUND}

Betatrophin is a member of the angiopoietin-like protein family (ANGPTL), and is also knowr as ANGPTL8, lipasin, RIFL (Refeeding-Induced Fat and Liver) and hepatocellular carcinomaassociated protein TD26. In human, betatrophin is predominantly produced by the liver, with a smaller amount produced by the adipose tissue. In mice, betatrophin has been shown tc significantly improve beta cell function and glucose tolerance. To date, we have evidence to support a relationship between betatrophin and insulin resistance (IR). Increased betatrophir concentrations have been shown in type 2 diabetes, obesity and gestational diabetes mellitus (GDM). During pregnancy, IR is known to increase due to the antagonistic effects of hormones produced by the placenta on insulin action; these hormones include: chorionic gonadotropin growth hormone, adrenocorticotropic hormone, placental lactogen, prolactin, estrogens and progestogens. Hyperplasia of beta cells prevents this IR from leading to the development of diabetes. Pregnancy is also a period of remarkably impaired lipid profiles; specifically, in both human and animal models, betatrophin has been shown to be involved in the regulation of lipid metabolism, especially triglycerides (TG)

\section{AIM}

The aim of our study was to evaluate the role of betatrophin in IR and function of beta cells during pregnancy in healthy women.

\section{SUBJECTS AND METHODS}

We examined 80 healthy pregnant women in each trimester (T1- first, T2-second, T3-third trimester), with 45 of the cohort also examined 3 months postpartum (3MPP). Our contro group comprised 30 non-pregnant healthy women $(\mathrm{HW})$ of reproductive age. We measured the levels of betatrophin (ELISA), glucose (enzymatic method with hexokinase), insulin (IRMA), C-peptide (EASIA), HbA1c (HPLC), and calculated HOMA-IR and HOMA\% $\beta$.

\begin{tabular}{|c|c|c|c|c|}
\hline \multicolumn{5}{|c|}{ RESULTS } \\
\hline & T1 $(n=80)$ & T2 $(n=80)$ & $(n=80)$ & 3 MPP $(n=45)$ \\
\hline Age (years) & $30.0(7.0)$ & & & \\
\hline BMI $\left(\mathrm{kg} / \mathrm{m}^{2}\right)$ & $\begin{array}{l}\text { Before pregnancy } \\
21.6(2.9)\end{array}$ & $\mathrm{N} / \mathrm{A}$ & $N / A$ & $23.2(4.0)$ \\
\hline Gestational age (week) & $11.0(3.0)$ & $21.0(3.0)$ & $33.0(2.5)$ & N/A \\
\hline Weight (kg) & $63.0(12.0)^{*}$ & $67.0(11.0)^{* *}$ & $74.8(11.5)^{* * *}$ & 64.0 (11.0) \\
\hline Weight gain (kg) & $1.9(2.8)^{*}$ & $5.5(3.8)^{* *}$ & $12.0(7.0)^{* * *}$ & $3.0(4.0)$ \\
\hline Betatrophin (ng/ml) & $1.8(1.5)^{*}$ & $1.5(1.3)$ & $1.2(1.3)^{* * *}$ & $1.5(1.5)$ \\
\hline Fasting glucose (mg/dl) & $83.0(8.5)$ & $80.0(8.0)$ & $80.0(7.0)^{* * *}$ & $87.0(7.0)^{* * * *}$ \\
\hline HbA1c (\%) & $4.9(0.4)$ & $4.8(0.4)$ & $4.9(0.4)^{* * *}$ & $5.2(0.4)^{* * * *}$ \\
\hline Fasting insulin ( $\mu \mathrm{lU} / \mathrm{ml})$ & $8.6(3.3)$ & $9.0(3.4)$ & $11.3(5.3)^{* * *}$ & $6.2(2.4)^{* * * *}$ \\
\hline C-peptide 0' (pmol/l) & $0.3(0.1)^{*}$ & $0.4(0.2)^{* *}$ & $0.5(0.2)^{* * *}$ & $0.4(0.2)$ \\
\hline HOMA-IR & $1.8(0.8)$ & $1.8(0.7)$ & $2.3(1.1)^{* * *}$ & $1.4(0.6)$ \\
\hline HOMA-B (\%) & $157.7(84.8)^{*}$ & $206.9(93.6)$ & $227.7(140.8)^{* * *}$ & $92.5(37.3)^{* * * *}$ \\
\hline Cholesterol (mg/dl) & $176.5(40.5)^{*}$ & $234.5(46.5)^{* *}$ & $262.0(52.0)^{* * *}$ & $198.5(49.5)$ \\
\hline LDL cholesterol (mg/dl) & $92.0(32.5)^{*}$ & $135.0(50.0)^{* *}$ & $162.0(59.0)^{* * *}$ & $110.0(50.0)^{* * * *}$ \\
\hline HDL cholesterol (mg/dl) & $70.0(17.5)^{*}$ & $83.5(18.5)$ & $79.0(20.0)^{* * *}$ & $70.0(19.0)$ \\
\hline Triglicerides (mg/dl) & $86.0(32.5)^{*}$ & $132.5(60.5)^{* *}$ & $193.0(83.0)^{* * *}$ & $59.5(38.0)$ \\
\hline TSH ( $\mu \mathrm{lU} / \mathrm{ml})$ & $0.8(0.9)^{*}$ & $1.4(0.8)$ & $1.3(1.0)$ & $1.2(0.8)^{* * * *}$ \\
\hline fT3 (pg/ml) & $3.2(0.4)^{*}$ & $2.9(0.4)$ & $2.9(0.4)$ & $2.9(0.4)^{* * * *}$ \\
\hline $\begin{array}{l}\text { fT4 }(\mathrm{ng} / \mathrm{ml}) \\
\text { Note: N/A-not applicable. Statisti } \\
*^{* *} \text { second (T2) and third trimeste } \\
* * * * \text { firct trimos }\end{array}$ & $\begin{array}{l}1.1(0.2)^{*} \\
\text { ically significant (P } \\
\text { er (T3); }{ }^{* * *} \text { third tr }\end{array}$ & $\begin{array}{l}1.0(0.1) \\
\text { differences betwee }\end{array}$ & $\begin{array}{l}0.9(0.1) * * * \\
: * \text { first (T1) and second t } \\
\text { postpartum (3MPP); }\end{array}$ & $\begin{array}{l}\text { :imester (T2) } \\
\text { : }\end{array}$ \\
\hline
\end{tabular}

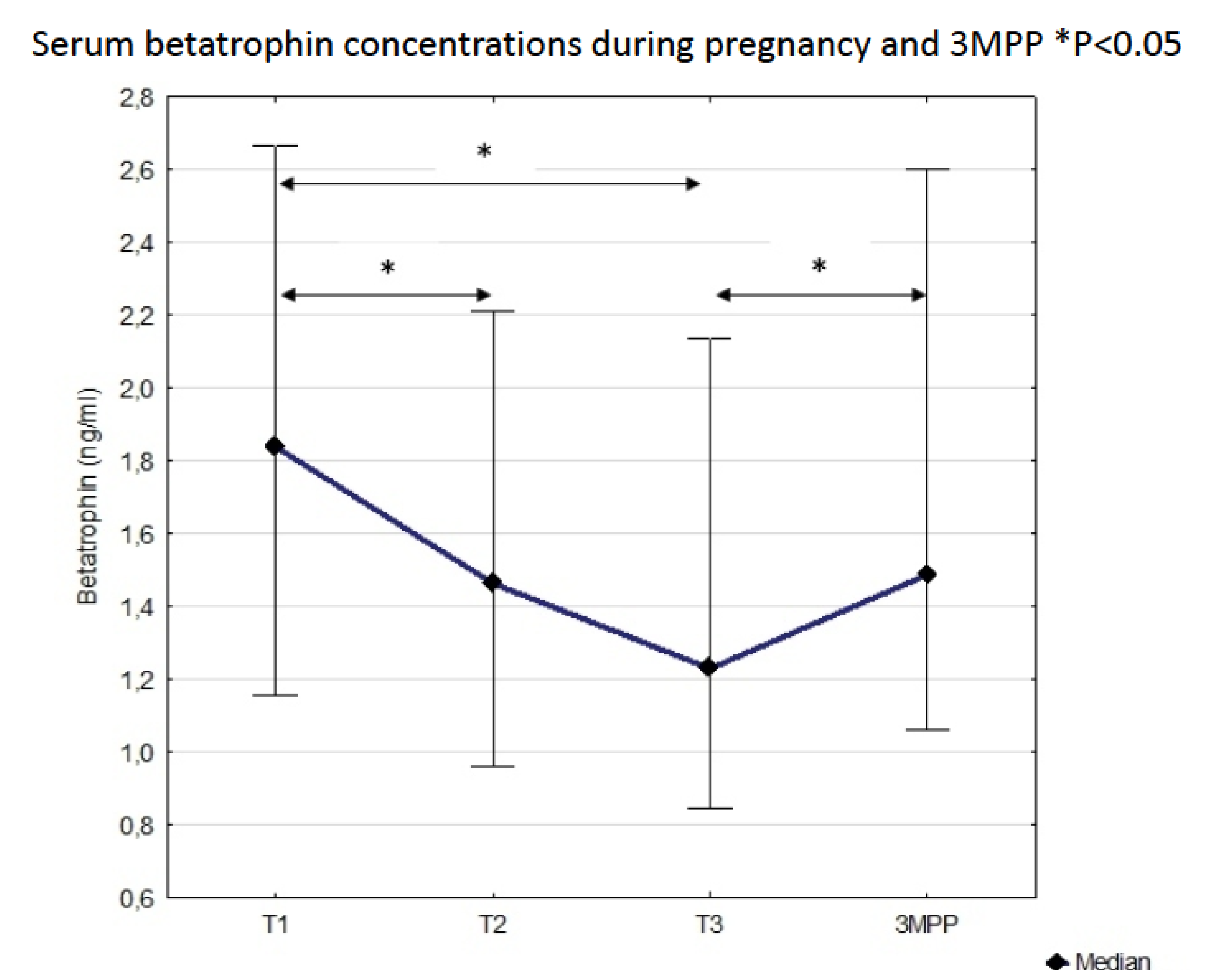

\begin{tabular}{|c|c|}
\hline & $3 M P P(n=45)$ \\
\hline Age (years) & $30.0(7.0)$ \\
\hline BMI $\left(\mathrm{kg} / \mathrm{m}^{2}\right)$ & $23.2(4.0)$ \\
\hline Weight (kg) & $64.0(11.0)$ \\
\hline Betatrophin (ng/ml) & $1.5(1.5)$ \\
\hline HbA1c (\%) & $5.2(0.4)$ \\
\hline C-peptide 0' (pmol/l) & $0.4(0.2)$ \\
\hline HOMA-IR & $1.4(0.6)$ \\
\hline HOMA-B (\%) & $92.5(37.3)$ \\
\hline Glucose $0^{\prime}(\mathrm{mg} / \mathrm{dl})$ & $87.0(10.0)$ \\
\hline Glucose $1 \mathrm{~h}$ (mg/dl) & $104.0(40.0)$ \\
\hline Glucose $2 \mathrm{~h}$ (mg/dl) & $91.0(26.0)$ \\
\hline Glucose AUC & $11460.0(3150.0)$ \\
\hline Insulin 0 ( $\mu \mathrm{IU} / \mathrm{ml})$ & $7.0(2.0)$ \\
\hline Insulin $1 \mathrm{~h}(\mu \mathrm{IU} / \mathrm{ml})$ & $35.6(31.6)$ \\
\hline Insulin $2 \mathrm{~h}(\mu \mathrm{IU} / \mathrm{ml})$ & $23.5(14.5)$ \\
\hline Insulin AUC & $3048.0(2271.0)$ \\
\hline $\mathbf{I S}_{120}$ & $8.5(3.7)$ \\
\hline $\mathrm{DI}_{\mathrm{OGTT}}$ & $2.2(1.0)$ \\
\hline Cholesterol (mg/dl) & $198.5(49.5)$ \\
\hline LDL cholesterol (mg/dl) & $110.0(50.0)$ \\
\hline HDL cholesterol (mg/dl) & $70.0(19.0)$ \\
\hline
\end{tabular}

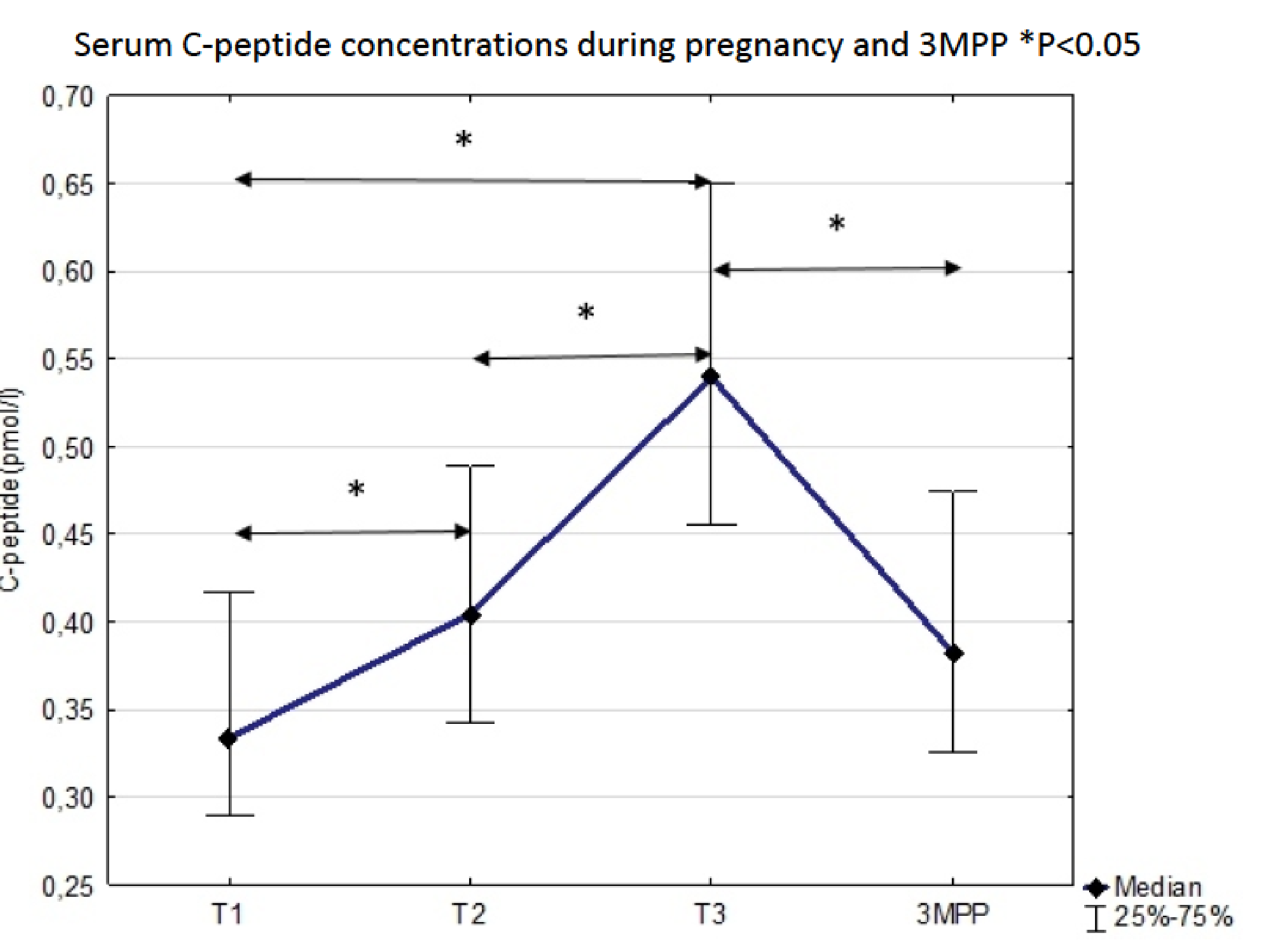

\begin{tabular}{ll} 
HW $(\mathrm{n}=30)$ & $\mathrm{p}$ value \\
\hline $33.0(14.0)$ & $\mathrm{Ns}$ \\
$22.1(4.5)$ & $\mathrm{Ns}$ \\
$62.0(11.0)$ & $\mathrm{Ns}$ \\
$1.5(1.8)$ & $\mathrm{Ns}$ \\
$5.2(0.3)$ & $\mathrm{Ns}$ \\
$0.5(0.2)$ & $\mathrm{p}=0.001$ \\
$1.5(1.3)$ & $\mathrm{Ns}$ \\
$88.9(68.0)$ & $\mathrm{Ns}$ \\
$86.0(6.0)$ & $\mathrm{Ns}$ \\
$121.5(51.0)$ & $\mathrm{Ns}$ \\
$94.5(19.0)$ & $\mathrm{Ns}$ \\
$12915.0(3840.0)$ & $\mathrm{Ns}$ \\
$6.7(6.1)$ & $\mathrm{Ns}$ \\
$35.5(30.6)$ & $\mathrm{Ns}$ \\
$21.6(31.4)$ & $\mathrm{Ns}$ \\
\hline $3060.0(3250.0)$ & $\mathrm{Ns}$ \\
\hline $8.3(7.1)$ & $\mathrm{Ns}$ \\
\hline $2.1(0.7)$ & $\mathrm{Ns}$ \\
\hline $183.0(55.0)$ & $\mathrm{Ns}$ \\
\hline $105.0(42.0)$ & $\mathrm{Ns}$ \\
$67.5(24.0)$ & $\mathrm{Ns}$ \\
\hline
\end{tabular}

\section{CONCLUSIONS}

HOMA-IR during pregnancy and 3 MPP $* \mathrm{P}<0.05$

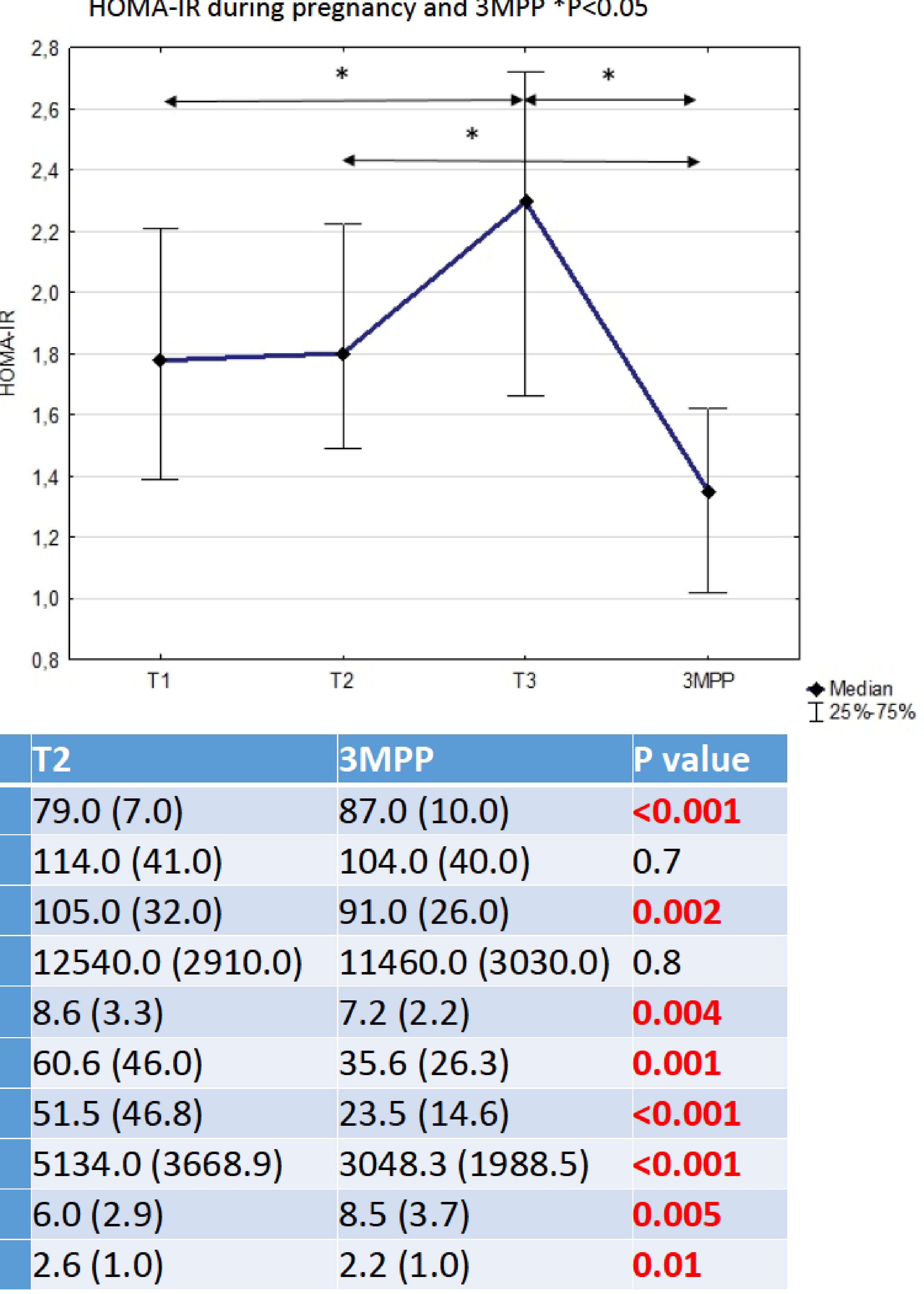

CORRELATIONS

\begin{tabular}{|c|c|c|c|c|}
\hline & T1 & T2 & T3 & 3MPP \\
\hline $\mathrm{DI}_{120}$ & ns & $r=0,25 p=0,004$ & ns & ns \\
\hline Weight & ns & ns & ns & $r=0,23 p=0,03$ \\
\hline $\begin{array}{l}\text { Weight } \\
\text { gain }\end{array}$ & $r=0,25 p=0,03$ & $r=0,27 \quad p=0,01$ & ns & ns \\
\hline Age & $r=-0,23 p=0,03$ & ns & ns & ns \\
\hline TSH & $r=0,24 p=0,03$ & ns & ns & ns \\
\hline $\mathrm{fT}_{4}$ & $r=-29 p=0,01$ & ns & ns & ns \\
\hline
\end{tabular}

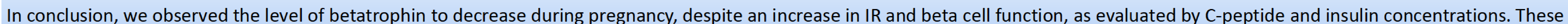

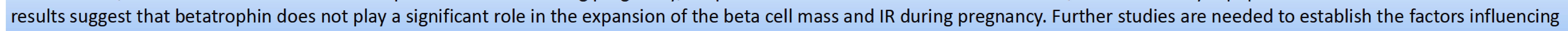
betatrophin levels during each trimester of pregnancy. 